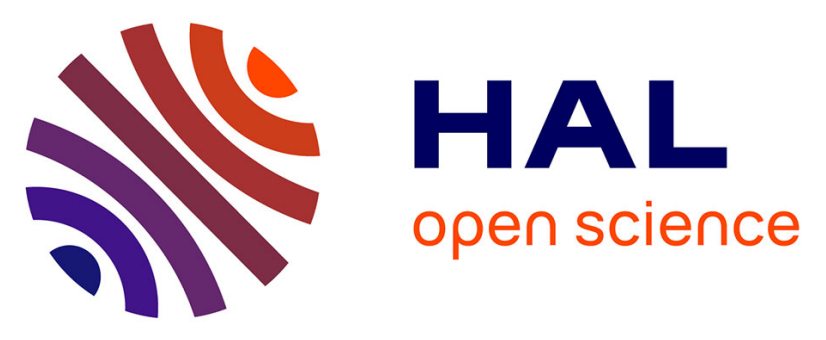

\title{
How Do Display Design and User Characteristics Matter in Animations? An Empirical Study with Air Traffic Control Displays
}

Sara Maggi, Sara Irina Fabrikant, Jean-Paul Imbert, Christophe Hurter

\section{To cite this version:}

Sara Maggi, Sara Irina Fabrikant, Jean-Paul Imbert, Christophe Hurter. How Do Display Design and User Characteristics Matter in Animations? An Empirical Study with Air Traffic Control Displays . ICC 2015, 27th International Cartographic Conference, Aug 2015, Rio de Janeiro, Brazil. pp.ISBN: 978-85-88783-11-9. hal-01202450

\section{HAL Id: hal-01202450 \\ https://hal-enac.archives-ouvertes.fr/hal-01202450}

Submitted on 2 Oct 2017

HAL is a multi-disciplinary open access archive for the deposit and dissemination of scientific research documents, whether they are published or not. The documents may come from teaching and research institutions in France or abroad, or from public or private research centers.
L'archive ouverte pluridisciplinaire HAL, est destinée au dépôt et à la diffusion de documents scientifiques de niveau recherche, publiés ou non, émanant des établissements d'enseignement et de recherche français ou étrangers, des laboratoires publics ou privés. 


\title{
How Do Display Design and User Characteristics Matter in Animations? An Empirical Study with Air Traffic Control Displays ${ }^{1}$
}

\author{
Sara Maggi, Sara Irina Fabrikant \\ Department of Geography / University of Zurich / Zurich / Switzerland \\ Jean-Paul Imbert, Christophe Hurter \\ ENAC / University of Toulouse / Toulouse / France
}

\begin{abstract}
We detail an empirical animation study to assess how display design and user spatial ability and training might influence visuospatial decision-making with animated displays showing aircraft movements. We present empirical results of a visuospatial detection task with moving objects, based on response accuracy and response time, including a descriptive eye-movement analysis. We found significant differences in a visuospatial detection task of moving objects across animation design types and domain expertise levels based on viewers' visuospatial skill differences. With this empirical approach, we hope to better understand how users explore and extract information from animated displays. Based on these results, we aim to further develop empirically validated animation display design guidelines to increase their efficiency and effectiveness for decision-making with and about moving objects.
\end{abstract}

Keywords: Animation, animation design, empirical cartography, expertise, training, spatial ability, eye tracker, air traffic control display

\section{RÉSUMÉ}

Nous présentons en détail une expérimentation évaluant des tâches utilisateurs dans un contexte de visualisation dynamique représentant des mouvements d'avions sur une interface de contrôle aérien. Nous cherchons à évaluer l'influence des principes d'affichage et du niveau d'expertise sur les aptitudes spatiales des participants à prendre des décisions. L'évaluation est basée sur l'exactitude des réponses données, le temps de réponse, et analyse descriptive des mouvements oculaires (eye tracking). Nous constatons des différences significatives dans la détection visuelle et spatiale d'objets en mouvement, selon le type d'animation et le niveau d'expertise des utilisateurs. À l'aide de cette expérimentation, nous espérons mieux comprendre comment les utilisateurs examinent, interprètent et extraient des connaissances à partir d'affichages dynamiques. À partir de ces résultats, nous souhaitons dégager des principes directeurs qui nous permettrons d'améliorer la conception d'animation, nous cherchons ainsi à accroître l'efficacité dans les prises de décisions des utilisateurs considérant la visualisation d'objets en mouvement.

Mots clés : Animation, animation cartographique, expérimentation empirique, expertise utilisateur, capacité visio-spatiale, oculométrie, contrôle aérien

\section{Introduction}

For the past 10 years, there has been a constant increase in the availability of movement data at high spatiotemporal resolution (Holyoak and others 2008). This trend is supporting research on new visual analytics methods to visualize and extract space-time information from these data (Andrienko and Andrienko 2008). Animated visualizations are to be considered as a tool facilitating the exploration of complex spatiotemporal phenomena, as demon- strated by the broad attention gained in the GIScience community (Andrienko and others 2010). Besides visuoanalytical capabilities, animated displays are also commonly used in the field of real-time surveillance. This is the case of air traffic control, where animated displays are employed in time-critical decision support with real-time movement data.

As Andrienko and others (2010) underline, a successful visual analytics tool needs to enable an efficient synergy with the perceptual and cognitive capabilities of the user. 
Intuitively, animations appear to be suitable to visualize and recognize spatiotemporal patterns (Moellering 1976). Real-world movement changes are depicted with changes over time being presented on the screen in a consistent way. This is in line with the "Congruence Principle," which emphasizes that effective graphics should be designed congruently and coherently according to users' mental principles (Tversky, Morrison, and Betrancourt 2002).

However, visual analytics research on dynamic visualization of movement data usually focus on the computational and technical aspect of extracting meaningful information from very large data sets (Klein, Van der Zwan, and Telea 2014). Very little attention has been paid to the understanding of the interaction between user characteristics and design of dynamic displays for decision support. The understanding of user reasoning and relevant information recognition currently faces a lack of empirical studies and sound cartographic design guidelines (Fabrikant and Lobben 2009; Shipley, Fabrikant, and Lautenschütz 2013). Making inferences based on animations of complex spatiotemporal information can be challenging because information is often too complex and presented too quickly to be processed simultaneously (Tversky, Morrison, and Betrancourt 2002). Hence, it imposes high perceptualcognitive demands on working memory (Lowe 1999) and this might hinder a user's attention on the detection and recognition of relevant movement patterns and movement behaviour for the task at hand.

Efficiency and effectiveness of visuospatial information processing with animated displays might be influenced by different factors, such as by external visual stimuli (e.g., display type, and the perceptual salience/thematic relevance of visual cues) and by viewer characteristics (e.g., expertise and spatial skills). Of particular interest is the question of how users with different skills identify and make decisions with animated displays showing anomalous or unexpected (real-time) movement patterns. Previous studies demonstrate that display design, as well as user background and training, can have a significant effect on task performance with graphical displays (Bartram, Ware, and Calvert 2001; Fabrikant and Goldsberry 2005; Kriz and Hegarty 2007). Moreover, users' spatial skills can considerably influence how information depicted on graphic displays is processed and acted upon (Wai, Lubinski, and Benbow 2009; Wright and others 2008).

To gain deeper insights on how humans perceive and process spatiotemporal phenomena on dynamic graphic displays, and on how cartographers should design animations according to perceptual and cognitive principles, we propose a long-term research framework to empirically assess and create general cartographic guidelines for perceptually salient, engaging, and cognitively inspired animations of movement data. Our research program is based on a holistic approach that couples eye movement data with electrodermal activity, electroencephalography, and traditional questionnaires (Maggi and Fabrikant 2014b).

Based on prior work, we present in this paper additional results of a human-subject experiment in the context of air traffic control (Maggi and Fabrikant 2014a). In particular, we aim to investigate how animation design (i.e., dynamic visual variables of the display), characteristics of the depicted objects (i.e., dynamics and complexity of the studied phenomenon), and user-related factors (i.e., individual differences and group characteristics of the users) might influence visuospatial information recognition with animations. We illustrate the statistical results of participants' behavioural analyses with animated displays (i.e., participants' response accuracy, response time, and eye movement measures) across expertise, participants' spatial skills, and animation design types. In the next section, we briefly review the state of the art concerning cartographic principles of animations, as well as cognitive and perceptual aspects of human visual attention.

\section{Related Work}

Since the 1930s, dynamic spatiotemporal phenomena and movement patterns have been increasingly visualized on dynamic cartographic displays, such as cartographic movies, including interactive $2 \mathrm{D}$ and $3 \mathrm{D}$ animations (Fabrikant 2005). The development of effective and efficient visual analytics methods of spatiotemporal phenomena has gained an increasing interest in GIScience in the past few years (Andrienko and Andrienko 2008; Andrienko and others 2010). However, the ways in which display design, displayed data, and users' characteristics might affect human perceptual and cognitive processes when viewing complex visuospatial animated displays is currently not well understood. It is not clear how people conceptualize movement change events and processes with animations or which animation design might be most appropriate for efficient and effective perception and interpretation of spatiotemporal phenomena (Battersby and Goldsberry 2010). Few human-subject studies with map animations have been conducted to date (Fabrikant and Goldsberry 2005; Harrower 2003).

Human reasoning with graphic displays is influenced by external representations and by internal processes (Hegarty, Kriz, and Cate 2003). Mental visualization capabilities, such as learning and reasoning about complex spatiotemporal phenomena, can be influenced by people's individual and group skills, and enhanced by adequate map design types (Fabrikant and Goldsberry 2005). In addition, mental visuospatial processes can be improved through training and practice (Wright and others 2008).

The proposed research study is based on cognitive and perceptual theories embedded in the field of cartography 
and vision science. For our study, three aspects of cartographic design and users' characteristics seem to be particularly relevant and challenging in developing effective and efficient animations:

- The cartographic principles of the animation design (i.e., the depiction of event dynamics and the visual variables of the display).

- The perceptual and cognitive aspects of the visual attention modulation with dynamic displays (i.e., the perceptual salience versus the thematic relevance of the visual cues, as well as the data and task complexity).

- The users' characteristics, such as people's expertise and spatial skills.

\section{CARTOGRAPHIC PRINCIPLES OF ANIMATION DESIGN}

To design dynamic displays of spatiotemporal events and phenomena adequately, a set of cartographic design guidelines, including dynamic visual variables, has been developed (DiBiase and others 1992; MacEachren 1995). Spatiotemporal information is depicted according to display moment, scene duration, scene frequency, frame order, rate of change between scenes, and synchronization of spatiotemporal phenomena. Scene frequency corresponds to the frame rate per second, and rate of change describes the amount of change between subsequent scenes. For example, a feature's position depicted on animated displays might be refreshed abruptly every four seconds or smoothly with a continuous animation. Fabrikant and Goldsberry (2005) suggest that smooth transitions between scenes might be effective for identifying changes between scenes, because they might reduce the change-blindness effect. However, smooth transitions might be also disadvantageous for users when they have to identify slight but relevant information changes embedded in a "noisy" background.

\section{PERCEPTUAL AND COGNITIVE ASPECTS OF DYNAMIC DISPLAYS}

Research on visual processing and on cartographic representations (Fabrikant and Goldsberry 2005) highlights that human visual attention is driven by bottom-up and top-down mechanisms. Visual cues in the visualization grab a viewer's attention from the bottom up. Considering perception principles of Gestalt theory (Koffka 1935), including relative motion patterns and the common fate principle, humans process visual scenes composed of different moving objects by grouping similar movement characteristics (e.g., objects with similar speed). Conversely, anomalous or dissimilar movement patterns depicted on a scene stand out and become perceptually more salient in a homogeneous background of similar movements (e.g., an object moving faster or at a different direction in a scene). Top-down processing of movement pattern recognition is driven by a viewer's cognitive mechanisms (e.g., a user's existing knowledge, expectations, and cognitive workload capacity). To study bottom-up and top-down visuospatial processing in an integrative fashion, the "NoticingSalience, Expectancy, Effort, and Value" (NSEEV) attention behaviour model has been developed based on studies on attentional processes with supervisory visualizations (Steelman, McCarley, and Wickens 2011). This method aims to predict the distribution of visual search and selective attention by considering the interaction between users' bottom-up (i.e., visual salience of events and effort) and top-down (i.e., expectancy of an event occurrence and value/importance of a task/event) processes in a dynamic environment. The NSEEV model highlights the importance of dynamic salience (i.e., motion and animation) in capturing visual attention. Imbert and others (2014) used this approach in the domain of air traffic control to assess the properties of several notification designs in dynamic radar visualizations.

The complexity of the animated display (e.g., the number of objects to be processed at the same time) and the viewers' expertise are relevant factors influencing viewers' bottom-up and top-down attentional processes. Boucheix and Lowe (2010) emphasize that unexperienced users (without any training with a specific visualization type or task) process animation displays based on perceptual salience, rather than on thematic relevance, as the visuospatial and temporal complexity of the animation increases. Humans use this approach as a strategy to minimize the increased processing demands. However, it is not currently clear how user expertise and training influence the perceptibility and recognition of task-relevant information in animations (Fabrikant 2005). In the domain of air traffic control, well-trained controllers may have to simultaneously process more than 20 aircraft, depending on the assigned air traffic space. However, in previous studies with continuous animations, users were able to process only a maximum of four moving objects at the same time (Ware 2013). Cavanagh and Alvarez (2005) argue that tracking more than four objects simultaneously is possible by multiple-object tracking (e.g., by grouping them according to their similar speed or direction). In addition, air traffic controllers can handle a larger amount of aircraft simultaneously by giving priority to the most relevant tasks (Niessen, Eyferth, and Bierwagen 1999).

\section{USER CHARACTERISTICS}

Regarding graphic displays, previous studies support the idea that not only map design, but also spatial skills and expertise might have an important effect on information processing with and information extraction from animations. For this reason, users' prior knowledge and expertise should be considered as well in designing animated displays (Kriz and Hegarty 2007). 
Spatial thinking and spatial skills have long been an important topic within psychology and cognitive science. They should have a similarly important role in information processing with animated displays, because the ways in which people process map information may differ according to their spatial abilities. Wai, Lubinski, and Benbow (2009) suggest that spatial ability might be a relevant factor in predicting future achievement in science, technology, engineering, and mathematics (STEM) domains. However, human spatial intelligence is not a biologically determined cognitive characteristic; it might be developed and improved by adequate training and practice (Wright and others 2008).

\section{Methods}

\section{USE CASE AND EXPERIMENTAL DESIGN}

To test how map design-, data-, and user-related factors might influence the apprehension span for task-relevant features of animations, we conducted a human subject study in the domain of air traffic control (ATC). Air traffic control displays are a well-suited use case for our experiment, because animations are a standard display type to monitor aircraft movements. The main goal of air traffic controllers is to monitor the air traffic space to ensure that aircraft maintain a minimal safety distance from each other, that the traffic flow is optimized to reduce delay and aircraft fly distance, and that anomalous or unexpected aircraft movement patterns are promptly detected. Air traffic controllers are exposed to stress situations on a daily basis, which presupposes significant cognitive workload and continuous processing of visuospatial information. This profession requires superior spatial skills. In fact, to enter the ATC training school, candidates have to pass specific spatial ability tests (e.g., tests on mental rotation and visuo-perceptual speed abilities).

Due to technical development over time, and thus historical reasons, standard air traffic control displays typically show aircraft movements using semi-static animations, in which aircraft positions are updated every four seconds (Hurter and Conversy 2008). It is possible to visualize continuous aircraft movements from GPS data (e.g., Flightradar $24^{2}$ ), but these data are not currently used at the operational level. Lee and Klippel (2005) suggest that aircraft movement changes visualized with continuous animated displays seem to be advantageous for controllers because task-relevant information is refreshed frequently compared to semi-static displays, and it might help them to create a better mental picture of the air traffic dynamics. Based on Tversky and others (2002)'s Congruence Principle, we would like to better understand how people detect movement changes within animations to respond to the following research questions: Are there differences in response efficiency and effectiveness between these two animation design types (i.e., semi-static vs. continuous animations)? Do these differences depend on the saliency of the depicted objects, as well as on movement dynamics and data complexity? Do these differences depend on user characteristics (i.e., individual and group differences)?

Our study uses a mixed-factorial design to answer these questions. We investigated how the independent variables animation design (i.e., semi-static vs. continuous animations), characteristics of the depicted objects (i.e., amount and relative speeds of the depicted objects), and participants' characteristics (i.e., ATC expertise and spatial ability) might influence the effectiveness and efficacy (i.e., response accuracy and response time) of dynamic information processing and aircraft movement detection.

\section{PARTICIPANTS}

In all, 37 participants took part in the experiment according to a between-subject design: 18 air traffic controllers at the Ecole Nationale de l'Aviation Civile (ENAC) in Toulouse (i.e., ATC experts) and 19 psychology students at Temple University in Philadelphia (i.e., ATC novices). The ATC experts are all well-trained air traffic controllers with more than 10 years of experience in the context of ATC. They are on average 38 years old; 16 ATC experts are men and two are women. ATC novices are students who have no specialist knowledge or training about ATC and are on average 22 years old. ATC novices are distributed equally in gender.

\section{MATERIALS}

We developed 16 ATC test stimuli according to the current French radar display system for air traffic control. The animations were created using Processing, ${ }^{3}$ a Java-based software program. Aircraft dynamics is based on real aircraft movement parameters (i.e., aircraft size, speed, and acceleration). Aircraft positions are represented by means of the Operational Display System (ODS) comet design (Figure 1). The ODS comet shows current and past aircraft positions with five squares of different size. The biggest square corresponds to the current aircraft position. Past positions are displayed with trailing squares of gradually smaller sizes. In addition, a speed vector is depicted as a line from the aircraft's current position to the predicted future position in a three-minute interval. Text labels located in proximity of the aircraft provide additional information to the air traffic controller (e.g., an aircraft's current speed and height, and aircraft type).

As mentioned earlier, we investigated two independent variables in our empirical study, including animation design and characteristics of the depicted objects. With respect to the independent variable animation design, we manipulated the dynamic variable scene frequency of the shown animated stimuli. According to a between-subject 


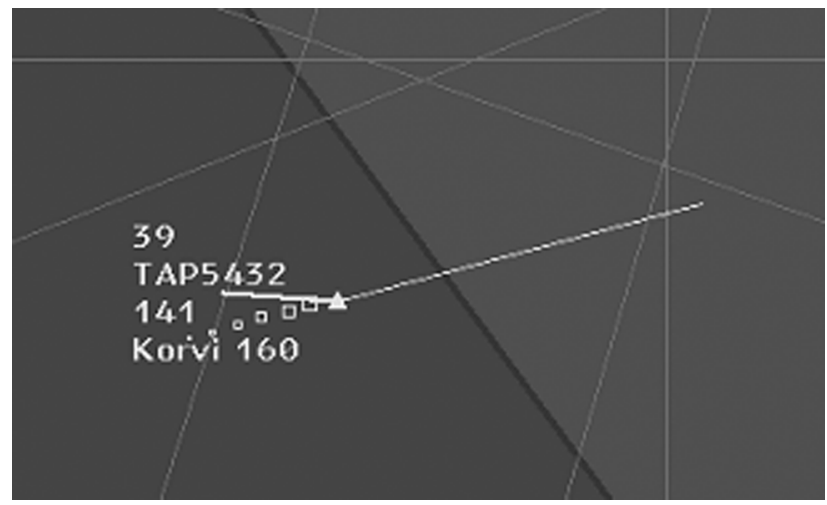

Figure 1. An aircraft represented on the French ATC radar display (ODS).

design, we developed 16 semi-static animations, in which aircraft positions refresh abruptly at four-second intervals (i.e., one frame each four seconds), and 16 continuous animations, in which aircraft positions refresh smoothly and continuously (i.e., 60 frames per second).

Regarding the independent variable characteristics of the depicted objects, we manipulated two factors: the number of visualized aircraft and their relative speeds. Eight of the 16 test stimuli show four aircraft, and eight displays include eight aircraft, respectively (Figure 2). We encoded four typical take-off speeds (160 kts [knots, or nautical miles per hour], $200 \mathrm{kts}, 250 \mathrm{kts}$, and $290 \mathrm{kts}$ ) in the animation. In four animations all aircraft move at the same speed (control displays), and in 12 displays aircraft move at different speeds. For all displays, all aircraft move at a constant speed throughout animation, except for one, which suddenly starts to accelerate. In displays where aircraft move at different speeds, the accelerating aircraft is never the fastest aircraft. We did this to investigate potential detection differences between thematically relevant information and perceptually more salient information. The accelerating aircraft changes its speed based on most common aircraft movements (e.g., A320), including an acceleration of $0.4 \mathrm{kts} / \mathrm{s}$. With semi-static animations, aircraft speeds and accelerations emerge from the screen by inferring changes between current and past positions of aircraft, as shown in Figure 3. Differences in speed and acceleration between aircraft can be inferred visually from the total length of the ODS radar comet and the spaces between its current and past positions (Figure 3). A fastermoving aircraft has greater spacing between the five position squares (see relationships of $\mathrm{d} 1$ and $\mathrm{d} 2$ in Figure 3), and thus a longer overall length (i.e., D in Figure 3 ) than a slowly moving aircraft. Similarly, an accelerating aircraft can be recognized by its constantly increasing spacing between the five position square (i.e., $\mathrm{d} 1$ increases more quickly than $\mathrm{d} 2$ in Figure 3 ) and consequently the constant increase in the total length of the comet (i.e., D in
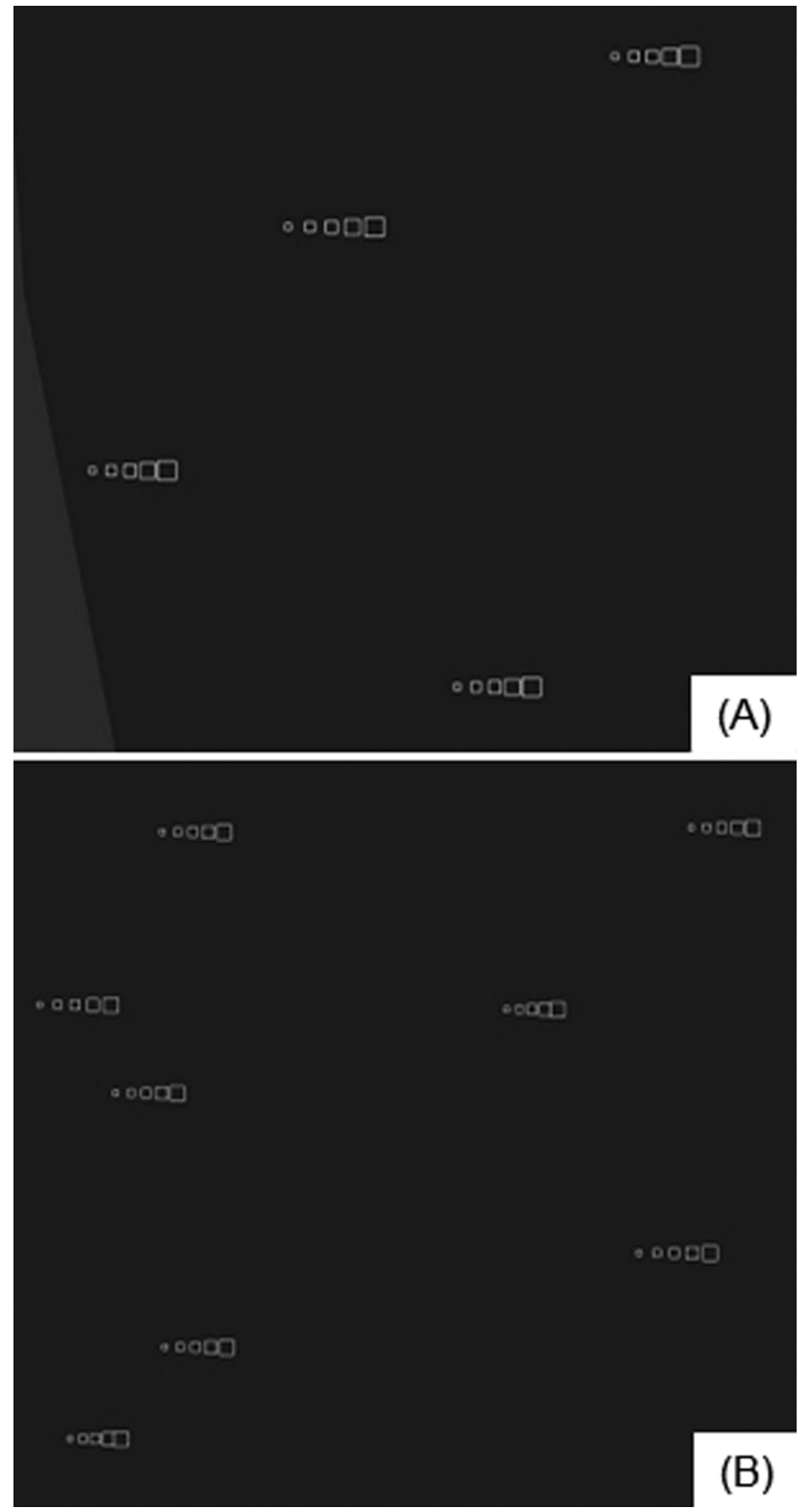

Figure 2. Static representation of two stimuli with four (A) and eight (B) aircraft moving from left to right on the screen at different speeds.

Figure 3; Hurter and Conversy 2008). With continuous animations, aircraft speeds and accelerations are perceived directly from the objects' motion across the display, while also being redundantly encoded graphically with aircraft position spacing and overall comet length. Acceleration is encoded as a constantly increasing velocity in the animation. Velocity changes are interpolated from the starting position and the ending position within the animation.

To evaluate only the effect of the mentioned independent variables, we kept all other potentially confounding variables constant (i.e., all aircraft move in the same direction), 


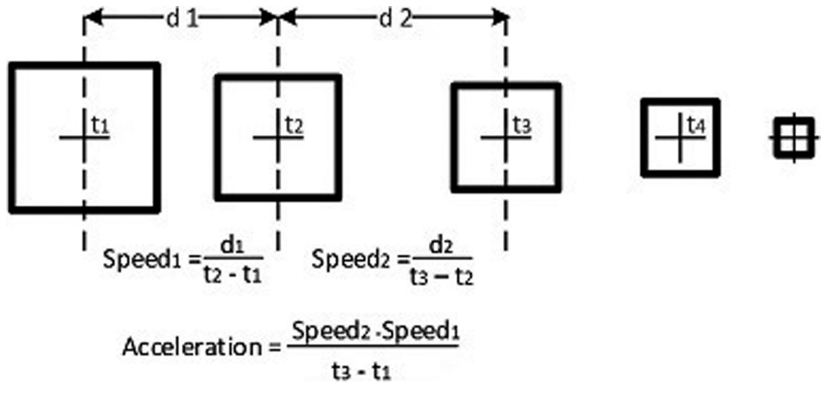

Figure 3. Speed and acceleration of an aircraft as depicted on the French ATC radar display (ODS) (Hurter, Conversy and Kapp 2008).

and they are depicted with the same colour hue and colour value (i.e., white aircraft moving on a homogeneous dark grey background). We also omitted text labels and speed vectors in order not to distract participants' attention from the motion behaviour of the comet-shaped aircraft.

\section{DATA COLLECTION AND TEST SET-UP}

As dependent variables, we recorded participants' response accuracy-that is, whether the task-relevant object was correctly identified or not-and task completion time. In essence, we recorded how much time participants took to identify the accelerating aircraft. We also measured participants' visual spatial skills using the Hidden Patterns Test, which measures humans' visuo-perceptual speed (Ekstrom and others 1976). This test is particularly suitable for assessing the speed and accuracy of visual search and scanning processes in a scene with specific objects hidden by other patterns. We recorded electrodermal activity with a skin conductance sensor ${ }^{4}$ and participants' brain activity with a mobile electroencephalogram. ${ }^{5}$ We also collected participants' eye movement data with a Tobii TX300 eye tracker. ${ }^{6}$ Eye tracking metrics might help us to assess individual and group differences on users' cognitive and perceptual processes. The statistical results of participants' brain activity and their skin conductance responses are not discussed in this paper due to space limitations, but they will be presented in a follow-up publication.

\section{PROCEDURE}

First, participants were asked to perform a Hidden Patterns Test to measure their spatial abilities. They were required to identify a specific figure that is hidden among other elements within six minutes. In all, they had to process 200 different patterns and mark whether a figure was visible or not. Next, after a brief training phase, according to the mentioned between-subject set-up, participants were asked to watch either 16 semi-static or 16 continuous animations depicting aircraft in motion. They were asked to detect the accelerating aircraft and click on it as soon as possible. Animations were presented to them digitally and in random order on a colour monitor at $1920 \times 1200$ spatial (pixel) resolution. Once participants identified the task-relevant aircraft, and confirmed their choice, the animation stopped. Following that, a set of five questions was presented to the participants to collect their response confidence, after which the next animation started. On average, participants took about 16 minutes to process the animated stimuli. Before and after being shown the animated displays, each participant filled in a Short Stress State Questionnaire (Helton 2004). Finally, participants were asked to fill in a post-test questionnaire to collect background information and judgements about the difficulty in solving the task. The obtained outcomes of users' effectiveness (task response accuracy) and efficiency (task response time), as well as users' spatial abilities differences and eye movement metrics across expertise and animation design, are elucidated in the next section.

\section{Results}

A subset of our results relating to participants' response accuracy, response time, and spatial abilities, including eye fixations across animation design types (i.e., semistatic vs. continuous animations) and ATC expertise levels (i.e., ATC experts and ATC novices), are described in this section.

\section{TASK RESPONSE ACCURACY}

As presented previously in Maggi and Fabrikant (2014a), we found that the mean response accuracy of ATC experts is close to $80 \%(M=81.44 \%, S D=15.51)$. This did not differ significantly across animation design conditions. Conversely, ATC novices performed the task less accurately than ATC experts for both animation types. However, this difference is significant only for the continuous displays $(F(1,17)=22.19, p<0.000)$, in which ATC novices performed the task with only $27.78 \%(S D=29.55)$ of correct responses. The response accuracy of ATC novices with continuous animations differs significantly from the accuracy of ATC novices with semi-static animations $(F(1,17)=$ $6.38, p<0.022)$, where $63.13 \%(S D=31.35)$ of them correctly identified the task-relevant object (i.e., the accelerating aircraft).

We further analyzed whether relative speed differences between aircraft might have an influence on participants' response accuracy across ATC expertise and animation design. We compared the response accuracy of the four stimuli depicting aircraft moving at the same speed (control displays), with the response accuracy of the 12 stimuli depicting aircraft moving at different speeds. As expected, participants perform the task significantly more accurately 


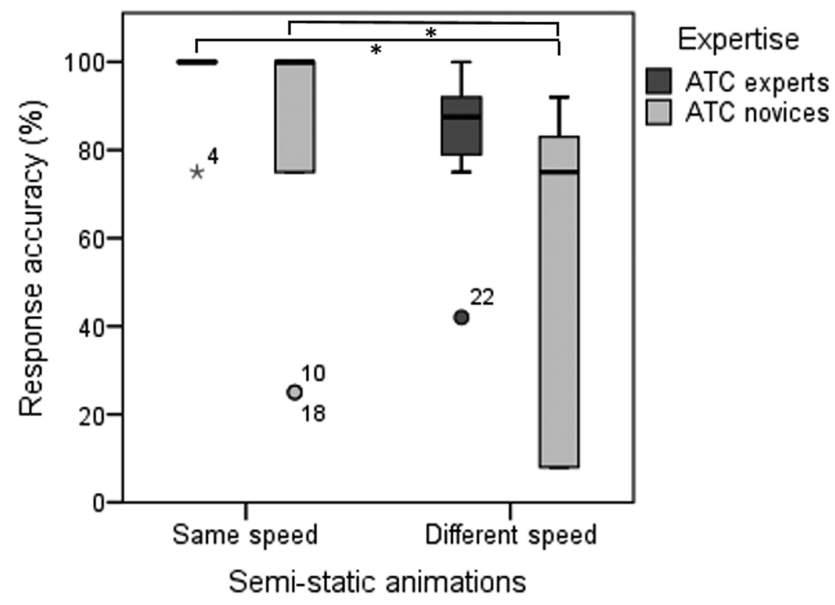

Figure 4. Response accuracy for ATC experts and ATC novices in the semi-static animation condition (same vs. different speeds, error bars show standard error).

with the stimuli displaying aircraft at the same speed $(\mathrm{Mdn}=100 \%)$ compared to those depicting aircraft moving at different speeds (Mdn $=67 \% ; Z=-4.79, p=$ 0.000 ). However, this difference is not significant between the two ATC expertise groups in the semi-static animation condition, as shown in Figure 4. In contrast, participants' accuracy is significantly different between ATC experts and ATC novices in the continuous animation condition. As can be seen in Figure 5, ATC experts performed the task more accurately than ATC novices with the control display $(Z=-2.65, p=0.008)$, as well as with the displays depicting aircraft at different speeds $(Z=-2.97, p=0.003)$. Moreover, in the continuous animation condition with aircraft moving at different speeds, the average response accuracy of ATC novices was $18.44 \%(S D=30.46$, Mdn $=$ $8 \%$ ), and this is barely above chance (i.e., $18.75 \%$ ). This significantly differs from their response accuracy with semistatic animations ( $\mathrm{Mdn}=75 \%, Z=-2.27, p<0.023)$. However, we did not find any significant difference of ATC novices' response accuracy with the control displays across the two animation design conditions.

We found that $66.92 \%$ ( $S D=22.23$ ) of the ATC novices and $21.67 \%(S D=15.86)$ of the ATC experts in the continuous condition $(F(1,22)=32.95, p<0.000)$ typically selected the perceptually more salient object (i.e., the fastest-moving object) rather than the task-relevant moving object (i.e., the accelerating aircraft). We further investigated this task performance difference across ATC expertise groups for the continuous animation condition by means of eye movement analysis and discuss this in the "Eye Movement Measures" section.

\section{TASK RESPONSE TIME}

We analyzed participants' task efficiency, considering task completion time of accurate responses only. We found a

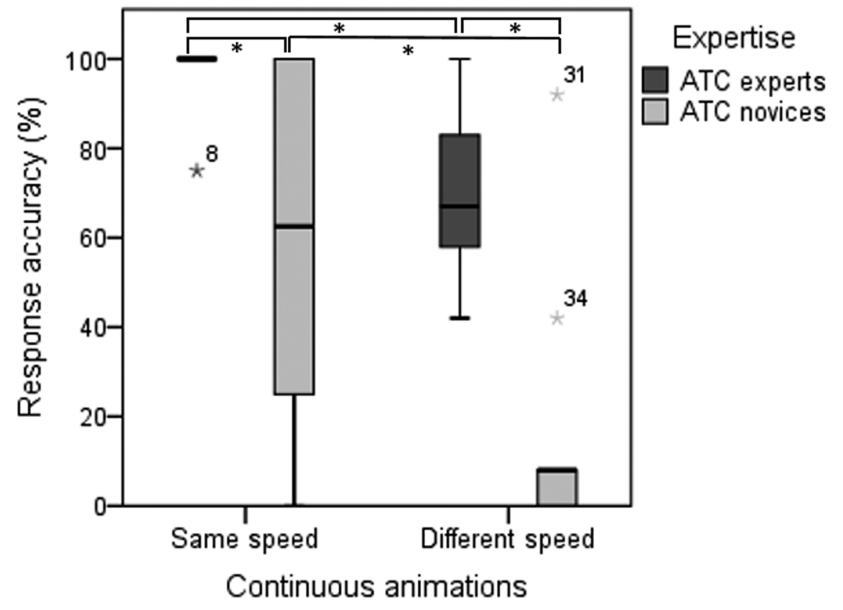

Figure 5. Response accuracy for ATC experts and ATC novices in the continuous animation condition (same vs. different speeds, error bars show standard error).

significant difference among ATC experts between the two animation design types $(F(1,16)=9.65, p<0.007)$. ATC experts performed the task faster with the semi-static animations $(M=46.50 \mathrm{~s}, S D=9.73)$ than with the continuous animations $(M=61.00 \mathrm{~s}, S D=9.93)$. This is in contrast to ATC novices, who performed the task with almost no difference in efficiency between the semi-static animations $(M=46.27 \mathrm{~s}, S D=16.69)$ and the continuous animations $(M=49.22 \mathrm{~s}, S D=20.51)$.

\section{SPATIAL ABILITIES}

We found a significant difference in spatial abilities across ATC experts and ATC novices $(F(1,35)=14.61, p<.001)$ according to the Hidden Pattern Test scores. ATC experts identified $66.94 \%$ ( $S D=11.48)$ of the test figures correctly, while ATC novices had a response accuracy of $48.05 \%(S D=17.74)$.

However, there is no correlation with spatial ability and the type of animation design. Furthermore, we computed a Pearson correlation of participants' response accuracy and spatial ability scores. As shown in Figure 6, overall, we find a moderate positive correlation between these two variables $(r=0.452, N=37, p<0.005)$.

We looked more closely at the data within the two animation display conditions and, as can be seen in Figure 7, found a stronger relationship between spatial abilities and participants' response accuracy with the continuous displays $(r=0.71, N=19, p<0.001)$. The Pearson correlation of the semi-static animations did not show any significant dependencies between these two variables.

\section{EYE MOVEMENT MEASURES}

To identify the perceptual and cognitive processes that supported participants' decision-making, we systematically 


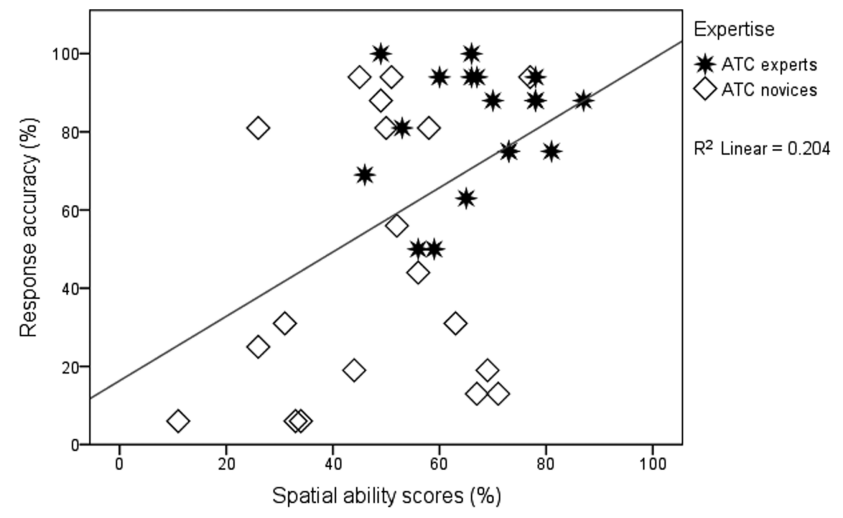

Figure 6. Correlation of participants' response accuracy and spatial abilities scores across ATC expertise (stars for ATC experts and diamonds for ATC novices).

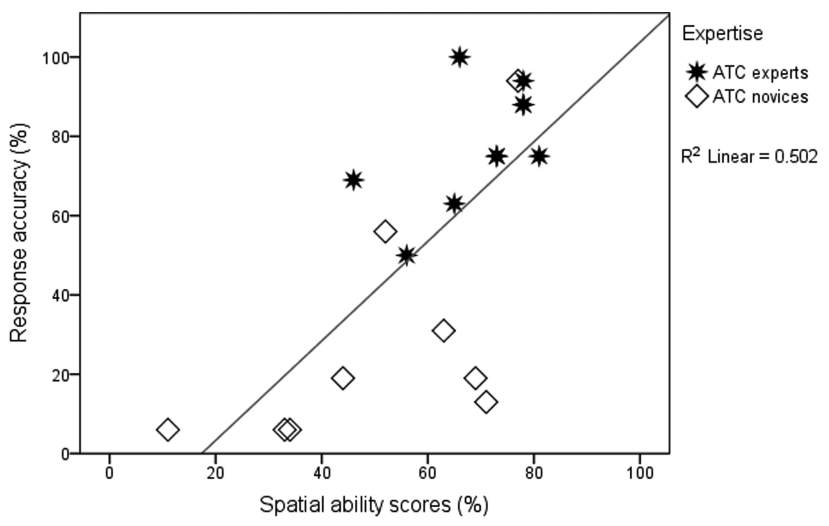

Figure 7. Correlation of participants' response accuracy and spatial ability scores for the continuous displays (stars for ATC experts and diamonds for ATC novices).

studied which aircraft participants focused on (i.e., area of interest, or AOI), for how long (i.e., eye fixation duration), and how frequently (i.e., eye fixation rate) across ATC expertise and animation design conditions by means of eye movement analyses. We employ common eye movement metrics for this (Fabrikant and others 2008).

We chose an I-VT classification algorithm to filter eye fixations with a minimum fixation duration of $60 \mathrm{~ms}$. We defined the depicted aircraft as AOIs and systematically assessed eye fixation durations and eye fixation rate (i.e., the number of fixations per AOI and per second) between the perceptually more salient aircraft (i.e., the fastest aircraft) and the task-relevant aircraft (i.e., the accelerating aircraft) for each stimulus. As response accuracy among ATC experts and ATC novices showed a significant difference for continuous animations, we analyzed participants' eye fixation durations for this design type only. Five of the 17 participants were not considered in the analysis because of insufficient quality of their eye movement data.

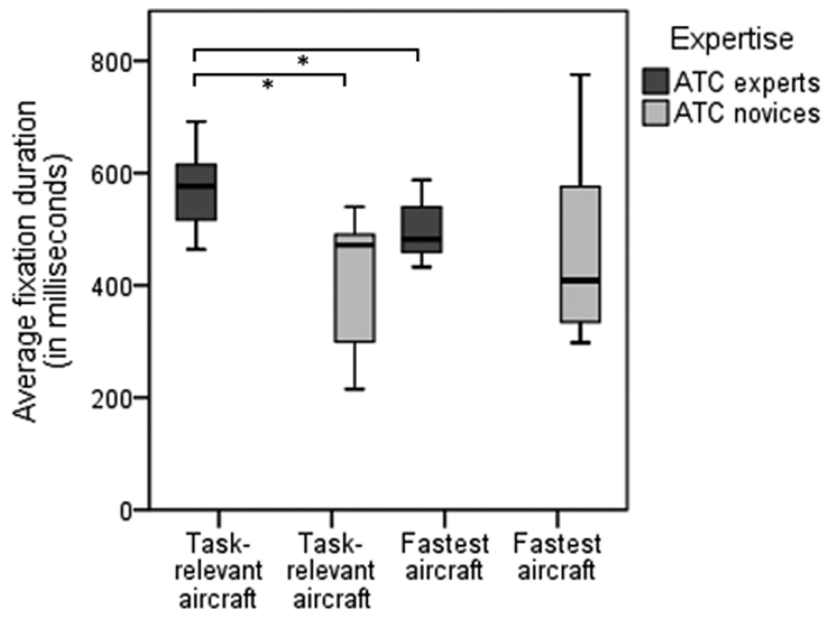

Figure 8. Average fixation duration of AOIs in continuous animations, for task-relevant (the two box plots on the left) and fastest aircraft (the two box plots on the right) across ATC expertise groups.

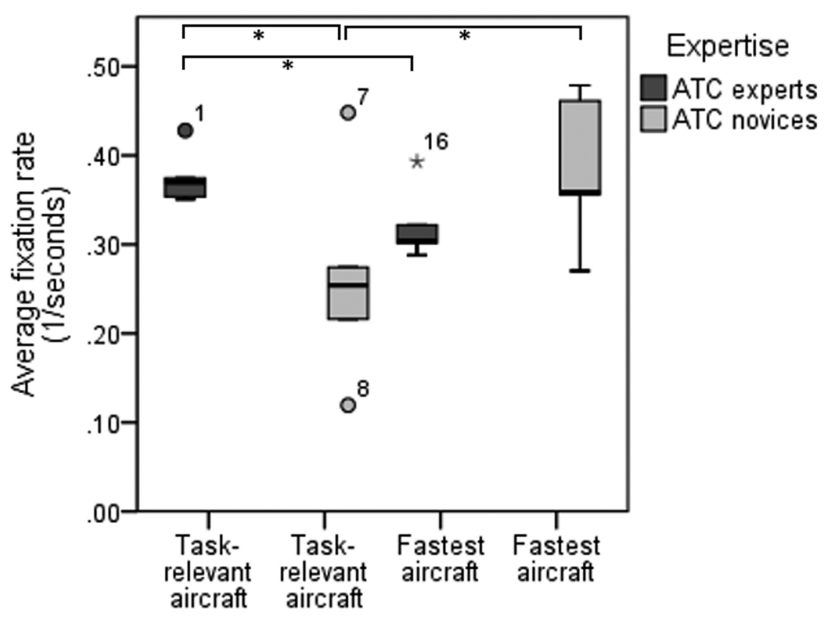

Figure 9. Average fixation rate of AOls in continuous animations, for task-relevant (the two box plots on the left) and fastest aircraft (the two box plots on the right) across ATC expertise groups.

We can observe a significant difference between the two ATC expertise groups in fixating task-relevant and perceptually more salient objects. On average, participants fixated all the aircraft depicted on the continuous animations for $484.82 \mathrm{~ms}(S D=119.90)$. As shown in Figure 8, ATC experts fixated both task-relevant $(M=573.47 \mathrm{~ms}$, $S D=78.69)$ and perceptually more salient aircraft $(M=$ $497.15 \mathrm{~ms}, S D=57.82$ ) for a significantly longer time than did ATC novices (task-relevant aircraft: $M=400.95$ $\mathrm{ms}, S D=128.71$; perceptually more salient aircraft: $M=$ $471.55 \mathrm{~ms}, S D=184.73 ; F(1,11)=6.60, p<0.026)$. ATC experts fixated the task-relevant objects for a signifi- 
cantly longer time compared to the average fixation duration $(t(12)=4.07, p<0.002)$. In contrast, ATC novices had significantly shorter fixation durations on the accelerating aircraft compared to the average fixation duration $(t(12)=-2.46, p<0.032)$.

The average eye fixation rate for all participants and all aircraft is $0.28 \mathrm{~s}^{-1}(S D=0.04)$. Overall, ATC experts $\left(M=0.29 \mathrm{~s}^{-1}, S D=0.02\right)$ fixated on aircraft slightly more frequently compared to ATC novices $\left(M=0.27 \mathrm{~s}^{-1}\right.$, $S D=0.05)$. However, as shown in Figure 9, the taskrelevant aircraft $\left(M=0.37 \mathrm{~s}^{-1}, S D=0.028\right)$ were fixated upon significantly more frequently by ATC experts than the fastest aircraft $\left(M=0.32 \mathrm{~s}^{-1}, S D=0.04 ; F(1,5)=\right.$ 8.57, $p<0.033)$. Conversely, ATC novices fixated on the fastest aircraft $\left(M=0.38 \mathrm{~s}^{-1}, S D=0.08\right)$ significantly more often than the task-relevant aircraft $\left(M=0.26 \mathrm{~s}^{-1}\right.$, $S D=0.11)(F(1,4)=24.17, p<0.008)$. ATC experts fixated on the task-relevant aircraft more frequently than ATC novices $(F(1,10)=6.28, p<0.031)$.

\section{Discussion}

In this empirical study, we investigated how animation design (i.e., frame rate per second), characteristics of the depicted objects (i.e., movement dynamics and complexity of the depicted objects), and user-related factors (i.e., ATC expertise and spatial skills) might influence visuospatial information detection with ATC animated displays. Our results suggest that animation design type, motion cues of the depicted moving objects, users' background, and spatial abilities do indeed influence visuospatial inference making with animations.

Overall, ATC novices performed the task less accurately than ATC experts. This difference is more pronounced for the novel continuous animations than for the semistatic animations. With this design type, response accuracy and eye fixation measures reveal that ATC novices focused their attention frequently on the fastest aircraft rather than on the task-relevant, accelerating aircraft. This finding confirms results of previous studies with continuous animations (Boucheix and Lowe 2010; Fabrikant 2005), in which participants processed information depicted on complex visuospatial displays according to perceptually salient features rather than to thematic relevant information. A possible explanation might be the perceptibility of a specific moving object (i.e., saliency of figure) in continuous animations according to its dynamic contrast strengths with the context in the background (Boucheix and Lowe 2010). In essence, this might be explained by the Gestalt principle of common fate (Koffka 1935). Elements that are in contrast with each other on a visual scene (e.g., objects moving faster compared to their nearby objects) are more easily distinguishable from their neighbours and the static background. As Boucheix and Lowe (2010) suggest, ATC novices explored information based on its perceptual salience with continuous animations because task-relevant information might be too hard to be correctly perceived, as it requires too much perceptual and cognitive processing demands. This outcome found a correspondence with the eye fixation measures between ATC expertise groups of the continuous animations as well. In contrast to ATC experts, ATC novices fixated more frequently upon the fastest aircraft than the accelerating aircraft. According to previous studies (Holmqvist and others 2011), a higher eye fixation rate on semantically informative areas should be indicative of the noticeability of those areas for a user. In addition, eye fixation durations of experts are generally longer than novices. A possible explanation is that experts process information more efficiently using a larger visual span due to increasing skills. Conversely, shorter fixations lead to reduced performance and might be an indicator of higher stress and cognitive workload (Henderson, Weeks, and Hollingworth 1999; Jacob and Karn 2003). An additional explanation for this difference between the two ATC expertise groups might be due to a different motivation in solving the task. Participants' answers from the post-test questionnaire showed that ATC novices, unlike ATC experts, judged the task not only as difficult, but also as boring. For this reason, we believe that, as a next step, it would be important and advantageous to couple these data with psycho-physiologic metrics to get a deeper insight about the participants' emotional and cognitive state (i.e., cognitive workload and motivation), and so to strongly validate our empirical results.

ATC experts performed accurately (at least $80 \%$ correct responses), regardless of animation design type. Their familiarity with the semi-static animated displays (due to training) and with the task allowed them to detect the accelerating aircraft despite the perceptual salience of thematically irrelevant information. The only significant difference that we found with ATC experts across animation designs is the time they took to respond. Not surprisingly, they took longer to respond with the novel continuous animations, as compared to the more familiar semi-static animations. This is probably due to their unfamiliarity with the continuous animation displays, and thus they needed more time to process the shown information. With continuous animations, the smooth transitions of the velocity changes of the accelerating aircraft might be as difficult to be effectively and efficiently perceived as for the ATC novices. Hence, recognition of the accelerating aircraft also becomes harder for them than with semi-static animations, and this is reflected in their response time.

Surprisingly, response accuracy of ATC novices in the semi-static animation condition did not significantly differ from that of ATC experts. This might be due to a differ- 
ence in perceptibility of the relative motion patterns across semi-static and continuous animation conditions. Velocity changes in the semi-static animations are not directly perceived as were the continuous animations. Participants had to infer speed differences and accelerations from the different aircraft shapes (i.e., participants can identify the fastest-moving aircrafts because of their elongated forms). That might reduce the dynamic contrast among the displayed aircraft and so prevent the erroneous detection of the perceptually more salient objects, rather than of the task-relevant information. Velocity changes in semi-static animations might be also perceived more effectively compared to continuous animations, because motion changes are represented as a sequence of discrete steps over time. This is in line with the event perception theories by Shipley and Zacks (2008). On one hand, continuous animations might be better suited for depicting movement data compared to semi-static animations, because continuous movement data are represented and perceived in a realistic and congruent way. On the other hand, with smooth and continuous transitions between scenes, it might be more difficult for users to effectively identify micro-step changes in aircraft velocity, compared to abruptly refreshing scenes, because it might require higher perceptual and cognitive demands on working memory.

Finally, as Newcombe and Frick (2010) suggest, people can be more successful in a particular academic or professional domain because of their spatial skills. As in the domain of air traffic control, operator candidates must possess good spatial skills to enter ATC training school. Their spatial skills will then probably improve during ATC training and their everyday job demands. This might explain the significant difference in spatial abilities across the two expertise groups in our experiment. However, even if we found a moderately positive correlation of participants' spatial abilities with response accuracy, this relationship might be relevant for the continuous displays only. Supposing that continuous animations require higher processing demands and higher cognitive load compared to semi-static animations, a higher spatial skill level might have a positive influence on completing the task effectively. This finding emphasizes the relevance of users' visuospatial skills and prior training on tested tasks, beyond display design. High spatial skills might be advantageous for people when processing information with novel (i.e., continuous) and even cognitively demanding animated displays. Training in a specific domain might help people in processing complex information and transferring previous knowledge onto unfamiliar and novel domains, even without significant compromise of task effectiveness.

\section{Summary and Outlook}

In this paper, we present results of an empirical study with animated map displays in an air traffic control (ATC) context. We investigate the influence of three factorsi.e., the animation design type (i.e., semi-static and continuous animations), the dynamics and contextual characteristics of the depicted moving objects (i.e., objects with same or different relative speeds), and user characteristics (i.e., ATC expertise and visual spatial skills) — on the effectiveness and efficiency of task-relevant visuospatial data processing with animations. We measured participants' response accuracy, task completion time, and spatial abilities with a Hidden Pattern Test, including participants' eye movements. Our results show that task performance with animations might be significantly influenced by the three factors identified (i.e., animation design, data, and user characteristics). Our findings confirm previous studies suggesting that display design, saliency, and relevance of the depicted moving objects, and users' prior knowledge and visual spatial skills, influence the effectiveness and efficiency of information processing with animations (Fabrikant 2005; Kriz and Hegarty 2007. Furthermore, we found that moving objects depicted with continuous animations are perceived differently compared to those represented with semi-static animations. Faster objects moving continuously on animated displays are perceived in a more salient way than those moving abruptly with a screen refresh rate of every four seconds. However, display design affects ATC novices more than ATC experts. Differences in familiarity, training, and participants' spatial skills do influence the effectiveness and efficiency of moving object detection with both semi-static and continuous animated display types.

As a next step, we would like to more deeply analyze the relationship between task performance and users' individual and group differences (e.g., cognitive load, motivation, and psycho-physiologic signals) with both animation design types by triangulating recorded eye movement sequences, electrodermal responses (i.e., galvanic skin conductance responses), and brain activity signals (i.e., EEG signals) of the participants. For example, we wish to investigate whether low performance of ATC novices could be due to higher mental workload or perhaps less motivation to solve the task. We also aim to further analyze eye movement sequences to identify participants' detection task strategies. Standard eye sequence analyses are not suited for our data due to their complexity (Krejtz and others 2014). A follow-up publication about these additional analyses is in preparation.

Follow-up experiments should be conducted with ATC experts to further investigate the influence of top-down processes. Furthermore, the effect of animation design in critical decision-making situations (e.g., detection and prediction of movement conflicts between aircraft) would be interesting. We hypothesize that appropriate visual variables employed for highlighting task relevant accelerations and transitions between actions and movement changes, or the inclusion of contextual/causal information 
(e.g., reference landmarks or weather information) in animated displays, might improve the detection of taskrelevant information (Lowe 2015). Enhanced visualization design should not only help experts, but also people with less training and lower visuospatial skills, to effectively guide their attention to task-relevant objects.

With our user-centred empirical studies, we hope to gain more insights on how emotional, cognitive, and perceptual processes might affect the effectiveness and efficiency of spatiotemporal data exploration depicted on dynamic displays. We further aim to develop sound cartographic design guidelines to create cognitively inspired and perceptually salient dynamic map displays that support effective and efficient visuospatial exploration of spatiotemporal phenomena.

\section{Acknowledgements}

This research was funded by the Swiss National Science Fund under grant no. 200020-134646. The authors wish to thank participants from the French Civil Aviation University (ENAC) and Temple University (USA) who kindly participated in our user study. We are also grateful to Maxime Cordeil at ENAC, including Tim Shipley and Kelly Bower at Temple, for their invaluable collaboration in the realization of the user study with ATC displays.

\section{Author Information}

Sara Maggi, MSc, is a geographer and a PhD student in the Geographic Information Visualization and Analysis Group (GIVA) of the Geographic Department at the University of Zurich (UZH), Switzerland. Her current research interests are in the field of dynamic cartography, cognitive and perception science, and psycho-physiologic responses to map design. Email: sara.maggi@geo.uzh.ch.

Dr. Sara Irina Fabrikant is a professor of Geography and currently Vice President of the International Cartographic Association (ICA). She has research interests in geovisual analytics, GIScience and cognition, and dynamic cartography. She is the appointed head of the Geography Department at the University of Zurich (UZH), where she also heads the Geographic Information Visualization and Analysis Group (GIVA) at the GIScience Center. Email: sara.fabrikant@geo.uzh.ch.

Dr. Jean-Paul Imbert is a research engineer at the Interactive-Computing Laboratory (LII) of the French Civil Aviation University (ENAC) in Toulouse, France. His research interests cover human computer interaction, adaptive automation, and situation awareness in the field of air traffic control. Email: jean-paul.imbert@enac.fr.

Dr. Christophe Hurter is professor at the InteractiveComputing Laboratory (LII) of ENAC in Toulouse, France. In 2014, he received his HDR (Habilitation à Diriger des Recherche) and in 2010 his PhD in Computer Science from the University of Toulouse. He is also associate researcher at the research centre for the French military Air Force (CReA), Salon de Provence, France. His research interests cover information visualization (InfoVis) and humancomputer interaction $(\mathrm{HCl})$, particularly the visualization of multivariate data in space and time. He also investigates the design of scalable visual interfaces and the development of pixel-based rendering techniques. He has been involved with several projects, including large data exploration tools, graph simplification (edge bundling), and paper-based interaction. Email: christophe.hurter@ enac.fr.

\section{Notes}

1. This paper significantly extends research presented in Maggi and Fabrikant (2014a).

2. Flightradar24 at http://www.flightradar24.com/

3. Processing at https://www.processing.org/

4. Smartband at http://www.bodymonitor.de

5. Emotiv EPOC by http://emotiv.com/

6. Tobii TX300 eye tracker at http://www.tobii.com/

\section{References}

Andrienko, G., N. Andrienko, U. Demsar, D. Dransch, J. Dykes, S.I. Fabrikant, M. Jern, M.-J. Kraak, H. Schumann, and C. Tominski. 2010. "Space, Time, and Visual Analytics." International Journal of Geographical Information Science 24 (10): 1577-600. http:// dx.doi.org/10.1080/13658816.2010.508043.

Andrienko, N., and G. Andrienko. 2008. "Supporting Visual Exploration of Massive Movement Data." In AVI 2008: Proceedings of the Working Conference on Advanced Visual Interfaces, Napoli, Italy, ed. S. Levialdi, 474-75. http://dx.doi.org/10.1145/ 1385569.1385663.

Bartram, L., C. Ware, and T. Calvert. 2001. "Moving Icons: Detection and Distraction." Proceedings of the IFIP TC.13 International Conference on Human-Computer Interaction (INTERACT 2001), Tokyo, Japan.

Battersby, S.E., and K.P. Goldsberry. 2010. "Consideration in Design of Transition Behaviors for Dynamic Thematic Maps." Cartographic Perspectives 65 (65): 16-32, 67-69. http:// dx.doi.org/10.14714/CP65.127.

Boucheix, J.M., and R.K. Lowe. 2010. "An Eye Tracking Comparison of External Pointing Cues and Internal Continuous Cues in Learning with Complex Animations." Learning and Instruction 20 (2): 123-35. http://dx.doi.org/10.1016/j.learninstruc.2009.02.015.

Cavanagh, P., and G.A. Alvarez. 2005. "Tracking Multiple Targets with Multifocal Attention." Trends in Cognitive Sciences 9 (7): 349-54. http://dx.doi.org/10.1016/j.tics.2005.05.009.

Medline:15953754

DiBiase, D., A.M. MacEachren, J.B. Krygier, and C. Reeves. 1992. "Animation and the Role of Map Design in Scientific Visualization." Cartography and Geographic Information Systems 19 (4): 201-14, 265-66. http://dx.doi.org/10.1559/ 152304092783721295.

Ekstrom, R.B., J.W. French, H.H. Harman, and D. Dermen. 1976. Manual for Kit of Factor-Referenced Cognitive Tests. Princeton, NJ: Educational Testing Service. 
Fabrikant, S.I. 2005. "Towards an Understanding of Geovisualization with Dynamic Displays." Proceedings of American Association for Artificial Intelligence (AAAl) 2005 Spring Symposium Series: Reasoning with Mental and External Diagrams: Computational Modeling and Spatial Assistance, Stanford University, Stanford, CA, 6-11.

Fabrikant, S.I., and A. Lobben. 2009. "Introduction: Cognitive Issues in Geographic Information Visualization." Cartographica 44 (3): 139-43. http://dx.doi.org/10.3138/carto.44.3.139.

Fabrikant, S.I., and K.P. Goldsberry. 2005. "Thematic Relevance and Perceptual Salience of Dynamic Geovisualization Displays." Proceedings of ICA/ACI International Cartographic Conference 2005, A Coruña, Spain.

Fabrikant, S.I., S. Rebich-Hespañha, N. Andrienko, G. Andrienko, and D.R. Montello. 2008. "Novel Method to Measure Inference Affordance in Static Small Multiple Displays Representing Dynamic Processes." Cartographic Journal 45 (3): 201-15. http://dx.doi.org/10.1179/000870408X311396.

Harrower, M. 2003. "Designing Effective Animated Maps." Cartographic Perspectives 44 (44): 63-65. http://dx.doi.org/10.14714/ CP44.516.

Hegarty, M., S. Kriz, and C. Cate. 2003. "The Roles of Mental Animations and External Animations in Understanding Mechanical Systems." Cognition and Instruction 21 (4): 209-49. http:// dx.doi.org/10.1207/s1532690xci2104_1.

Helton, W.S. 2004. "Validation of a Short Stress State Questionnaire." Proceedings of the 48th Meeting of the Human Factors and Ergonomics Society 48 (11): 1238-42. http://dx.doi.org/ $10.1177 / 154193120404801107$.

Henderson, J.M., P.A. Weeks, and A. Hollingworth. 1999. "The Effects of Semantic Consistency on Eye Movements during Complex Scene Viewing." Journal of Experimental Psychology. Human Perception and Performance 25 (1): 210-28. http:// dx.doi.org/10.1037/0096-1523.25.1.210.

Holmqvist, K., M. Nyström, R. Andersson, R. Dewhurst, H. Jarodzka, and J. van de Weijer. 2011. Eye Tracking: A Comprehensive Guide to Methods and Measures. Oxford: Oxford University Press.

Holyoak, M., Casagrandi, R., Nathan, R., Revilla, E., and O. Spiegel. 2008. "Trends and Missing Parts in the Study of Movement Ecology." Proceedings National Academy of Sciences 2008, 19060-65. http://dx.doi.org/10.1073/pnas.0800483105.

Hurter, C., and S. Conversy. 2008. "Towards Characterizing Visualization." In Interactive Systems. Design, Specification, and Verification: Lecture Notes in Computer Science 5136, ed. T.C. Graham and P. Palanque, 287-93. Berlin: Springer. http://dx.doi.org/10.1007/978-3-540-70569-7_26.

Hurter, C., S. Conversy, and V. Kapp. 2008. "An Infovis Approach to Compare ATC Comets." 3rd International Conference on Research in Air Transportatoin ICRAT 2008. Fairfax, VA, USA.

Imbert, J.P., H.M. Hodgetts, R. Parise, F. Vachon, F. Dehais, and S. Tremblay. 2014. "Attentional Costs and Failures in Air Traffic Control Notifications." Ergonomics 57 (12): 1817-32. http:// dx.doi.org/10.1080/00140139.2014.952680. Medline:25202855

Jacob, R.J.K., and K.S. Karn. 2003. "Eye Tracking in Human Computer Interaction and Usability Research: Ready to Deliver the Promises." In The Mind's Eye: Cognitive and Applied Aspects of Eye Movement Research, ed. J. Hyönä, R. Radach, and H. Deubel, 573-605. Amsterdam: Elsevier. http://dx.doi.org/10.1016/B978044451020-4/50031-1.
Klein, T., M. Van der Zwan, and A. Telea. 2014. Dynamic Multiscale Visualization of Flight Data." Proceedings of VISAPP 2014.

Koffka, K. 1935. Principles of Gestalt Psychology. New York: Harcourt.

Krejtz, K., T. Szmidt, A.T. Duchowski, and I. Krejtz. 2014. "EntropyBased Statistical Analysis of Eye Movement Transitions." Proceedings of Eye Tracking Research and Applications Symposium (ETRA). Association for Computing Machinery, 159-66. http:// dx.doi.org/10.1145/2578153.2578176.

Kriz, S., and M. Hegarty. 2007. "Top-Down and Bottom-Up Influences on Learning from Animations." International Journal of Man-Machine Studies 65 (11): 911-30.

Lee, P.U., and A. Klippel. 2005. "Dynamic Aspects of Spatial Information in Air Traffic Controller Displays." AAAl Spring Symposium: Reasoning with Mental and External Diagrams: Computational Modelling and Spatial Assistance 2005, 18-23.

Lowe, R.K. 1999. "Extracting Information from an Animation During Complex Visual Learning." European Journal of Psychology of Education 14 (2): 225-44. http://dx.doi.org/ 10.1007/BF03172967.

Lowe, R. 2015. "Perceptual Learning in the Comprehension of Animations and Animated Diagrams." In The Cambridge Handbook of Applied Perception Research (2 vols.), ed. R. Hoffman, P. Hancock, M. Scerbo, R. Parasuraman, and J. Szalma, 692-710. New York: Cambridge University Press. http://dx.doi.org/10.1017/ CB09780511973017.042.

MacEachren, A.M. 1995. How Maps Work. Representation, Visualization, and Design. New York: Guilford.

Maggi, S., and S.I. Fabrikant. 2014a. "Embodied Decision Making with Animations." Proceedings of International Conference on Geographic Information Science 2014, Vienna, Austria.

Maggi, S., and S.I. Fabrikant. 2014b. "Triangulating Eye Movement Data of Animated Displays." Proceedings of International Workshop on Eye Tracking for Spatial Research 2014, Vienna, Austria, 1241: 27-31.

Moellering, H.M. 1976. "The Potential Uses of a Computer Animated Film in the Analysis of Geographic Patterns of Traffic Crashes." Accident, Analysis and Prevention 8 (4): 215-27. http:// dx.doi.org/10.1016/0001-4575(76)90007-5.

Newcombe, N.S., and A. Frick. 2010. "Early Education for Spatial Intelligence: Why, What, and How." Mind, Brain and Education: The Official Journal of the International Mind, Brain, and Education Society 4 (3): 102-11. http://dx.doi.org/10.1111/j.1751228X.2010.01089.x.

Niessen, C., K. Eyferth, and T. Bierwagen. 1999. "Modelling Cognitive Processes of Experienced Air Traffic Controllers." Ergonomics 42 (11): 1507-20. http://dx.doi.org/10.1080/ 001401399184857. Medline:10582037

Shipley, T., S.I. Fabrikant, and A.-K. Lautenschütz. 2013. "Creating Perceptually Salient Animated Displays of Spatially Coordinated Events." In Cognitive and Linguistic Aspects of Geographic Space: New Perspectives on Geographic Information Research, Springer Lecture Notes of Geoinformation and Cartography, ed. M. Raubal, D.M. Mark, and A.U. Frank, 259-70. Berlin: Springer. http://dx.doi.org/10.1007/978-3-642-34359-9_14.

Shipley, T.F., and J.M. Zacks. 2008. Understanding Events: From Perception to Action. New York: Oxford. http://dx.doi.org/ 10.1093/acprof:oso/9780195188370.001.0001. 
Steelman, K.S., J.S. McCarley, and C.D. Wickens. 2011. "Modeling the Control of Attention in Visual Workspaces." Human Factors 53 (2): 142-53. http://dx.doi.org/10.1177/0018720811404026. Medline:21702332

Tversky, B., J.B. Morrison, and M. Betrancourt. 2002. "Animation: Can It Facilitate?" International Journal of Human-Computer Studies 57 (4): 247-62. http://dx.doi.org/10.1006/ijhc.2002.1017.

Wai, J., D. Lubinski, and C.P. Benbow. 2009. "Spatial Ability for STEM Domains: Aligning Over 50 Years of Cumulative Psychological Knowledge Solidifies its Importance." Journal of Educational Psychology 101 (4): 817-35. http://dx.doi.org/10.1037/ a0016127.
Ware, C. 2013. Information Visualization: Perception for Design. San Francisco: Morgan Kaufmann.

Wright, R., W.L. Thompson, G. Ganis, N.S. Newcombe, and S.M. Kosslyn. 2008. "Training Generalized Spatial Skills." Psychonomic Bulletin \& Review 15 (4): 763-71. http://dx.doi.org/10.3758/ PBR.15.4.763. Medline:18792502 